\title{
PERSPECTIVE
}

\section{Big Data As an Enabler of Primary Education}

\author{
Vasant Dhar, Nandan Nilekani, Shankar Maruwada, and Nagaraju Pappu ${ }^{4}$
}

The journey of a thousand miles begins with one step.

-Lao Tzu

Tens of millions of children across the globe lack a basic primary education and are unable to exercise their full potential as citizens and participate as productive members of society. This is arguably one of the worst shortcomings of the human condition. We are addressing this problem of early childhood learning through the EkStep platform, ${ }^{*}$ which is currently under development in India. "EkStep" means "one step" in Hindi.

We realize that there are no easy solutions, but we might have answers if we think about education in new ways. We have learned a lot through the successes and failures of various government initiatives in many countries and "Edtech" solutions that attempt to improve some aspect of education. A common lesson is that we must simultaneously consider the pedagogical, technological, and cultural issues to address the critical constraints that hinder basic learning. In this article, we identify these constraints and explain how they may be addressed, specifically by considering the role of machines and "big data" to augment traditional processes of education.

The EkStep platform leverages the accessibility of smartphones and mobile devices that are becoming increasingly common in the world. The platform provides the pedagogic digital infrastructure for learning that includes fundamental concepts in numeracy and literacy. These concepts are the foundation to further learning. The platform also anchors analytics for content creation, tutoring, assessment, and reporting. All these functions are available programmatically through Application Programming Interfaces. The platform is "open" and designed to allow large-scale crowd-sourced collaboration, curation, and assembly

${ }^{*}$ www.ekstep.org of relevant content in multiple languages. The latter capability is especially important in India considering its linguistic diversity.

The conventional solution to the problem would be to train millions of gifted teachers over the next decade and place them in schools across the country that are equipped with state-of-the-art facilities. This is wishful thinking. We cannot wait for 10 years considering the tens of millions of children who desperately need help today, nor do we have the resources to create physical facilities and train large numbers of well-paid and qualified teachers.

Our solution is to integrate technology creatively into the learning process by addressing the scarce resources involved, namely expertise, time/attention, and varied content. This is not about delivering paper-based learning through a new medium, but about using the new medium's unique strengths to create new types of engagement and more learning opportunities by addressing the three constraints explicitly. For example, touchbased mobile devices, unlike personal computers, do not require a literate person to interact with them. Unlike a personal computer, the experience in a touch-based mobile device is naturally intuitive and multimedia, so that a child does not need to know how to read, write, or have technology awareness to interact with the device-playing with it using simple touch-based gestures makes the device come alive. Ironically, a machine also has the sociological advantage of being nonjudgmental, basing its advice and actions on data it ingests through its listening, speaking, and responding functions. Indeed, expert human teachers do much the same, but such teachers are in very short supply.

Expertise is clearly a scarce resource, as is the time or attention that can be allocated to each child when it is 
needed the most. Teachers have expertise but they have limited time and attention to devote to individual students or to prepare tailored content. In contrast, parents, especially mothers who typically spend the most time with their children after school and can supervise their homework, usually lack the expertise and cannot provide critical inputs when needed. Private tutors are often used after school, but they are not cheap and have limited time to customize content to each pupil.

Expertise and attention play a critical role in the various phases of learning, which consists of a series of "Instruction, Practice, and Assessment" cycles. Learning depends on the number of learning opportunities, in effect, the number of such cycles and the quality of assistance available in each cycle. Children vary considerably in terms of their listening, speaking, reading, and skills, which a skilled teacher recognizes to tailor assistance accordingly. Could a machine learn to do this effectively?

The short answer is yes in theory, but it will take some effort to design such machines. How might we do so? Fortunately, many of the basic concepts covered in primary education have been well researched and defined, which provides a good basis for evaluation. Numeracy, for example, involves a few dozen concepts that cover basic arithmetic operations, relationships, and shapes.

Literacy is somewhat more complex, involving phonology and visual representation of combinations of symbols known as orthography. Every language has a set of symbols, sounds, and a "threshold vocabulary" that serve as a basis for expression. Indian languages use compound conjugations and modifications of consonants and vowels compared with the linear left to right structure of English. This additional compositional complexity provides an unambiguous correspondence between the visual and the phonetic. Figure 1 shows two similar sounding words in Telugu-"sthree" (woman) and "shree" (auspicious)-where the base consonant " $\mathrm{s}$ " is modified twice in the first case (with a soft " $t$ " and an " $r$ ") and the base consonant "sh" is modified once in the second case (with the " $r$ "), and the vowel " $\mathrm{e}$ " is stretched as in "eel" in both cases. In addition to the compounding of symbols, Indian languages also allow relatively free word order at the sentence level, meaning there is no "one right" order for a sentence.

Learning, both in numeracy and literacy, involves abstraction and formation of new concepts. Recognizing when abstraction has occurred is a key aspect of evaluation. A skilled teacher can recognize a child's zone of proximal comfort in real time and recognize what to do next. For example, knowing when and how to take a child from concrete shapes and objects to numbers is key to assisted learning. Similarly, progressing from a picture and its name to the letters and word corresponding to the association is an abstraction that a skilled teacher knows how to recognize or facilitate. How might such a capability be learned by a machine on its own?

Fortunately, the mobile device is very interactive and generates a rich trail of plentiful telemetry data from each gesture and touch. The photographs (Fig. 2) and Supplementary Videos show children interacting with mobile devices individually and in groups, and facilitators engaging children through the platform (Supplementary Data are available online at www.liebertpub .com/big).

The telemetry data provide a powerful basis for learning. Imagine a world where the machine observes millions of children interacting with content through mobile devices. The data contain a rich and dense set
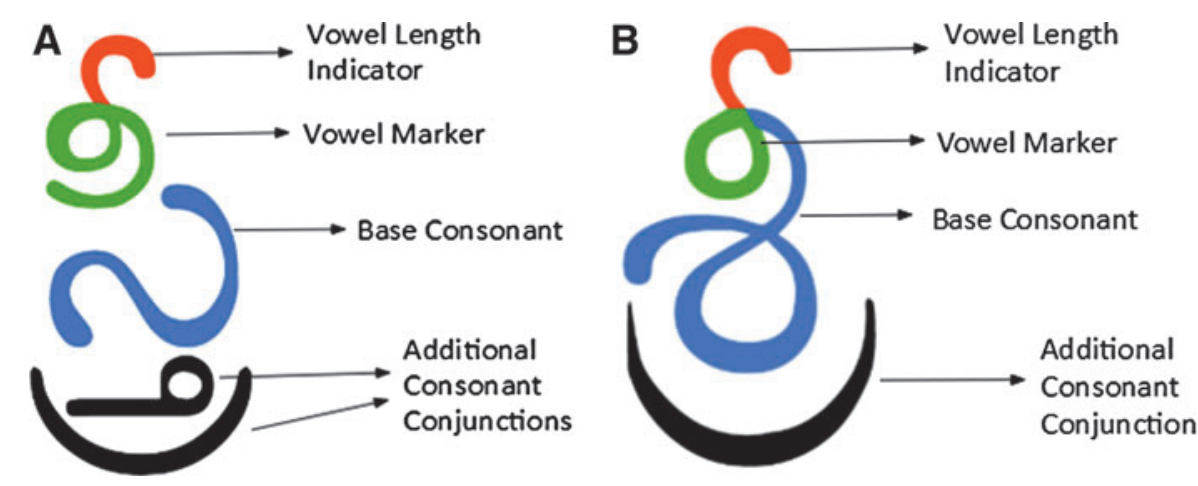

FIG. 1. (A) A complex syllable in Telugu for "sthree." (B) A complex syllable in Telugu for "shree." 

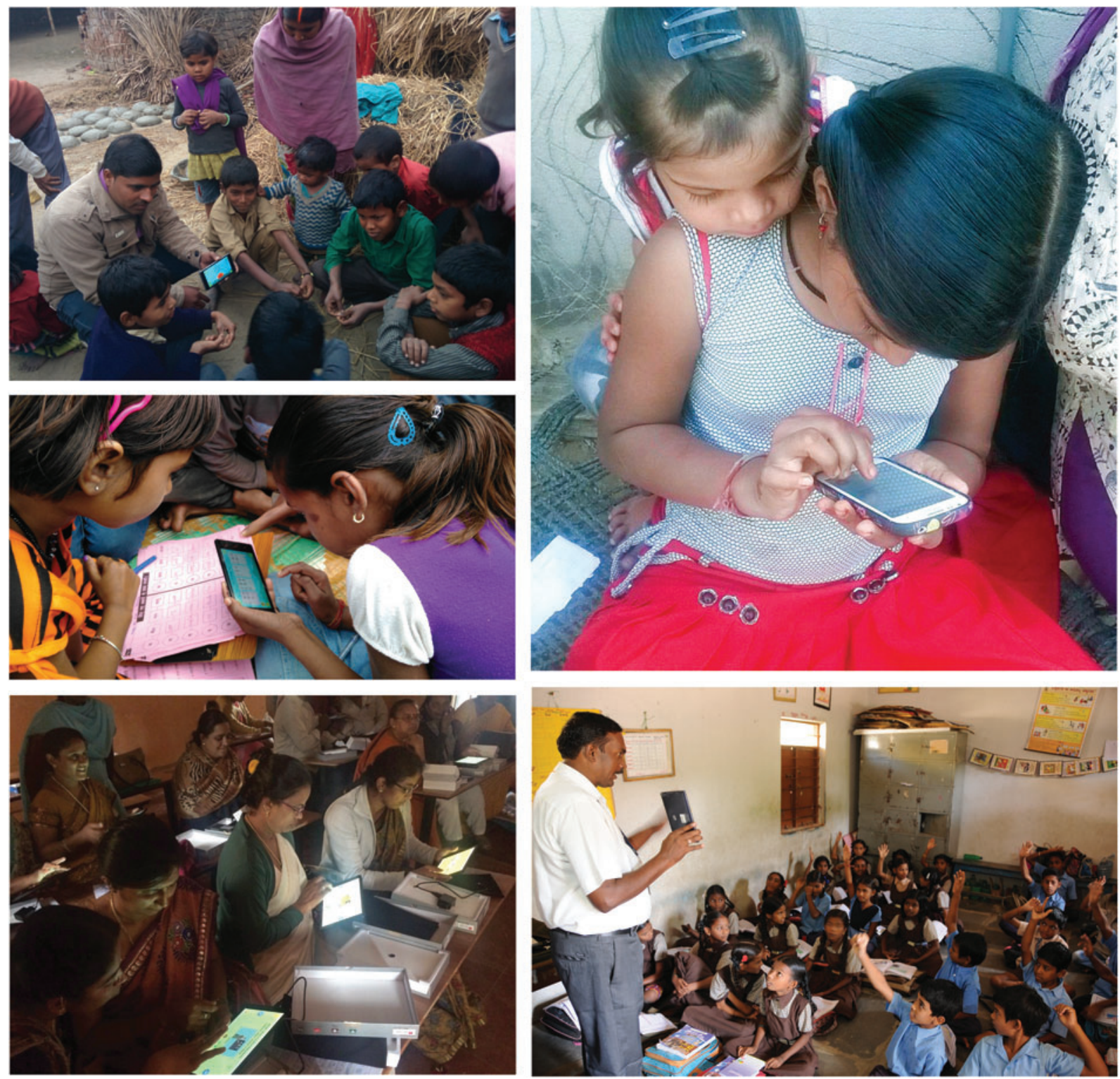

FIG. 2. Clockwise from top left: volunteers using smartphones at a remedial reading camp in rural Bihar, India; girl immersed with device while sister peeps; teacher engaging with device in classroom; teacher training session in Karnataka; students in a hybrid-learning setting.

of temporal observations of children playing games, solving problems, reading, writing, listening, and watching. One can imagine that this type of data gathered through neutral observation or controlled experiments could yield actionable generalizations. A machine might ask itself, for example, when does listening to a recorded audio improve the child's ability to pronounce a word correctly? Does a better understanding of fractions make it easier to understand relations? What is a good way to know whether a generalization has occurred with respect to some concept? The list of potential questions is virtually limitless. How does the machine find the useful ones?

The field of machine learning is about enabling machines to ask the right questions of the data, where answers constitute useful knowledge if they satisfy specific criteria. For example, high predictive accuracy might be one such validation criterion, as in "for children under 
$\mathrm{X}$ years of age, listening to an audio clip of a word $\mathrm{N}$ times improves pronunciation accuracy by $\mathrm{Y}$ percent, based on $\mathrm{Z}$ million observations." This ability enables the machine to be adaptive and capable of absorbing ever increasing amounts of data that lead to discoveries, and improve its knowledge base. Over time, there is no theoretical impediment to the machine achieving levels of assistance where expertise and attention are no longer scarce.

In a series of popular television commercials, the State Farm insurance company featured the leader in basketball assists, Chris Paul, to remind us that outcomes ultimately depend on how well players assist each other. It is no different in education, where assisted learning kick starts self-learning until the next assist is required. The right assist at the right time maximizes the number of learning or "scoring" opportunities. This capability is intended to be at the heart of EkStep platform both for learners and content curators. Indeed, the EkStep platform is "open" in the sense that it is designed to support crowd-sourced curation in multiple languages. The platform will provide tags to enable teachers and curators to build effective hints and "scaffolding" in the content to enable learning and its measurement. Measurement, in turn, allows the platform to tailor recommendations and scaffolding to each child.

The open platform coupled with a machine-learning capability also addresses the scarcity of good digital content, which is expensive to create and hard to do well. The platform provides a community with the "primitives" required to create content in its native language in a way that is additive to its knowledge base. In literacy, for example, imagine a roughly equivalent threshold vocabulary in every language in which content will be organized. The machine could learn to find the best threshold vocabulary on the basis of user experiences. The platform can also assist creators with tagging content appropriately, thereby finding its fit within the existing knowledge base. Google's various crowd-sourced tools such as Maps are a good analogy in that they allow users to add information about specific geographic locations or objects.

We believe that success will ultimately depend on using humans and machines in combination, exploiting the strengths of each. The enabler is data, the availability of which is transforming the role of machines in many areas of human activity by giving them the ability to learn the right things at the right time automatically from the data. Artificial intelligence and machine learning have advanced to a point where they are making possible entirely new realities by enabling machines to learn useful things from data that we might not even have envisioned. There is no reason to believe that it will be any different in education where we believe the big bang for the buck is in scaling up scarce resources, namely, expertise, individualized attention, and varied content. Sociologically, the education challenge is about how machines can support and help humans (caring adults) to increase access to learning opportunities for their children or the children in their community. It is clear to us that the cost of creating the EkStep digital platform is tiny in the larger scheme of things and the potential benefits are large compared with the status quo or business as usual.

Cite this article as: Dhar V, Nilekani N, Maruwada S, Pappu N (2016) Big data as an enabler of primary education. Big Data 4:3, 137-140, DOI: 10.1089/big.2016.29011.vdc. 